\title{
AN ANISOTROPIC INTEGRAL OPERATOR IN HIGH TEMPERATURE SUPERCONDUCTIVITY
}

\author{
BORIS MITYAGIN \\ Dedicated to Plamen Diakov on the occasion of his sixtieth birthday
}

\begin{abstract}
A simplified model in superconductivity theory studied by P. Krotkov and A. Chubukov [KC1, $\mathrm{KC} 2$ led to an integral operator $K$ - see (1), (2). They guessed that the equation $E_{0}(a, T)=1$ where $E_{0}$ is the largest eigenvalue of the operator $K$ has a solution

$(*)$

$$
T(a)=1-\tau(a) \text { with } \tau(a) \sim a^{2 / 5}
$$

when $a$ goes to $0 . \tau(a)$ imitates the shift of critical (instability) temperature.

We give a rigorous analysis of an anisotropic integral operator $K$ and prove the asymptotic $(*)$ - see Theorem 8 and Proposition 10.

Additive Uncertainty Principle (of Landau-Pollack-Slepian [SP], [LP1, LP2]) plays important role in this analysis.
\end{abstract}

0. Many models of high temperature superconductivity [LV] lead to the family of integral operators with anisotropic kernels. Structure and spectral analysis of these operators could be difficult and quite interesting because standard analytical methods (perturbation theory, Fourier transform, etc.) do not necessarily help us.

P. Krotkov and A. Chubukov [KC1, KC2] [see [KC2], section B.2, (46)-(60)] simplified one of local Eliashberg gap equations by dropping the Matsubara frequency summation and came to the operator in $L^{2}(\mathbb{R})$

$$
K \equiv K_{a T}: f(x) \rightarrow \int_{-\infty}^{\infty} K(x, y) f(y) d y
$$

where

$$
K(x, y)=\frac{1}{\pi} \cdot \frac{1}{T^{2}+(x-y)^{2}+a^{2}\left(x^{2}+y^{2}\right)^{2}} .
$$

2000 Mathematics Subject Classification. Primary 45C05; Secondary 35P05, $47 \mathrm{~B} 34$.

Key words and phrases. eigenvalues, spectra, anisotropic integral operators, instability temperature in superconductivity. 
If $a=0$ the operator is a convolution

$$
k * f \quad \text { where } k(x)=\frac{1}{\pi} \cdot \frac{1}{T^{2}+x^{2}}
$$

and its Fourier transform $\widetilde{k}(s)=\frac{1}{T} e^{-T|s|}$. Therefore, $\left\|K_{0 T}\right\|=1 / T$,

$$
\left\|K_{01}\right\|=1
$$

and its spectrum $\sigma\left(K_{01}\right)=[0,1]$ or $\sigma\left(K_{0 T}\right)=[0,1 / T]$. If $a>0$, $K(x, y)=K(y, x)>0$,

$$
\iint K^{2} d x d y<\infty
$$

so $K$ is a self-adjoint Hilbert-Schmidt operator, its spectrum $\left\{E_{n}(a)\right\}$ is discrete and by monotonicity

$$
\left\|K_{a} 1\right\|<1
$$

Subspaces $H^{e}$ and $H^{o}$ in $L^{2}(\mathbb{R})$ of even and odd functions are invariant so we'll consider restrictions

$$
K^{e}=K \mid H^{e} \quad \text { and } \quad K^{o}=K \mid H^{o}
$$

and their spectra. Let $E^{e}(T, a)$ and $E^{o}(T, a), a>0$ be the largest eigenvalues of $K^{e}$ and $K^{o}$, correspondingly. By (3) and (6)

$$
\begin{aligned}
& E^{e}(a, 1)=1-\varphi(a), \varphi>0, \\
& E^{o}(a, 1)=1-\psi(a), \psi>0,
\end{aligned}
$$

and $E^{o}(a, T)=1$ for some

$$
T=1-\tau(a), \tau>0 .
$$

The toy model in [KC2], Sect B.2, views $\tau(a)$ as an imitation of the shift of critical (instability) temperature where $a$ is the dimensionless quantity proportional to both the curvature and interaction (see (36), (37) in [4] for details). Heuristic manipulations (46)-(62) in [KC2 intended to make us believe that $\tau(a) \sim c a^{2 / 5}$. Maybe, this is quite remarkable that the potential " $2 / 5$ " appears in this analysis. We will show in this essay that indeed for $a>0$ small enough

$$
c_{1} a^{2 / 5} \leq \tau(a) \leq c_{2} a^{2 / 5}
$$

where $c_{1}, c_{2}>0$ are absolute constants (see Prop. 10 in Sect 4 below).

1. But first, we will find good estimates of the shift $\psi$ in (9), i.e., the behavior of the largest eigenvalue of $K^{o} \in(7)$. Of course,

$$
E(a)=\left\|K_{a}\right\| ; E^{e}(a)=\left\|K_{a}\left|H^{e}\left\|; E^{o}=\right\| K_{a}\right| H^{o}\right\|
$$


because $K$ is a self-adjoint compact operator and $H^{e}, H^{o}$ are its invariant subspaces.

Lemma 1. For $a>0$

$$
1>E(a)=E^{e}(a)>E^{o}(a)
$$

and

$$
E(0)=E^{e}(0)=E^{o}(0)=1 .
$$

Proof. As (3) shows, after Fourier transform

$$
\widetilde{K}_{01}=\mathcal{F}^{-1} K_{01} \mathcal{F}
$$

is a multiplier-operator

$$
\widetilde{k}: \varphi(s) \rightarrow e^{-|s|} \varphi(s)
$$

and

$$
\langle\widetilde{k} \varphi, \varphi\rangle=\int_{-\infty}^{\infty} e^{-|s|}|\varphi(s)|^{2} d s
$$

(13) follows easily. But $\widetilde{K}_{01}$ is not compact and the norms in (13) are not attained as values of a quadratic form

$$
\langle K f, f\rangle, \quad\|f\|=1, f \in H^{e} \text { or } H^{o} .
$$

Or if you wish they are "attained" if $f^{e}=\delta(x)$ and $f^{o}=\delta^{\prime}(x)$.

If $a>0$, the norm is attained as, say,

$$
E^{o}(a)=\left\langle K_{a} g, g\right\rangle, \exists g \in H^{o},\|g\|=1
$$

where $K_{a} g=E^{o}(a) g$. But then

$$
g_{*}(x)=|g(x)| \text { is even, }\left\|g_{*}\right\|=\|g\|=1,
$$

and

$$
E^{e}(a) \geq\left\langle K g_{*}, g_{*}\right\rangle>\langle K g, g\rangle=E^{o}(a) .
$$

We have a strict inequality in (20) because $K(x, y)>0$ and an odd $g(x) \neq 0$ is negative and positive on some subsets of positive measure. So

$$
\iint_{\mathbb{R}^{2}} K(x, y) g(x) g(y) d x d y<\int_{\mathbb{R}^{2}} K(x, y)|g(x)| \cdot|g(y)| d x d y .
$$

Indeed, if $g$ is odd and not identically zero put

$$
\begin{aligned}
& G^{+}=\left\{x \in \mathbb{R}^{1} \mid g(x)>0\right\}, \\
& G^{-}=\left\{x \in \mathbb{R}^{1} \mid g(x) \leq 0\right\} .
\end{aligned}
$$


Then measures $\lambda\left(G^{+}\right)>0, \lambda\left(G^{-}\right)>0$ are positive and

$$
\iint_{\mathbb{R}^{2}} K(x, y) g(x) g(y) d x d y
$$

$=$ sum of four integrals $\iint_{G^{ \pm} \times G^{ \pm}}$. Two of then (over $G^{+} \times G^{+}$and $G^{-} \times G^{-}$) are positive and two (over $G^{+} \times G^{-}$and $G^{-} \times G^{+}$) are negative because $K(x, y)>0$ everywhere with the excess being equal to

$$
-\left(\int_{G^{+} \times G^{-}} K g(x) g(y) d x d y\right)>0 .
$$

Almost the same argument shows that $E(a)<1$. Indeed,

$$
K_{a}(x, y)<K_{0}(x, y)
$$

everywhere on $\mathbb{R}^{2}$. So

$$
E(a)=\left\langle K_{a} g^{*}, g^{*}\right\rangle
$$

for some $g^{*} \in L^{2}\left(\mathbb{R}^{1}\right),\left\|g^{*}\right\|=1, g^{*} \geq 0$, and (22) implies

$$
E(a)<\left\langle K_{0} g^{*}, g^{*}\right\rangle \leq\left\|K_{0}\right\|=1 .
$$

Lemma 1 is proven.

2. From now on we analyze the integral operator $K=K_{a}, a>0$, with a kernel (2), $T=1$. By (17) we can consider it in the block-form

$$
K \sim\left[\begin{array}{cc}
K^{e} & 0 \\
0 & K^{o}
\end{array}\right]
$$

where $K^{e}, K^{o}$ are integral operators but their kernels are not uniquely determined because, say for $h \in H^{o}$ and $A(x, y)=A(x,-y)$

$$
\int A(x, y) h(y) d y=0
$$

any way. To analyze $K^{o}$ we change a kernel (2) to its antisymmetrization

$$
K^{\prime}(x, y)=\frac{1}{2}[K(x, y)-K(x,-y)
$$

and still have a representation

$$
K^{o} f=\int K^{\prime}(x, y) f(y) d y, \forall f \in H^{o} .
$$

Of course,

$$
\int K^{\prime}(x, y) g(y) d y=0, \forall g \in H^{e}
$$

this is a twin of (26). 
Explicit formula for $K^{\prime}$ is

$$
\begin{aligned}
K^{\prime}(x, y)=\frac{1}{\pi} & {\left[\frac{1}{1+(x-y)^{2}+a^{2}\left(x^{2}+y^{2}\right)^{2}}-\right.} \\
& \left.-\frac{1}{1+(x+y)^{2}+a^{2}\left(x^{2}+y^{2}\right)^{2}}\right]= \\
& =\frac{1}{2 \pi} \cdot \frac{4 x y}{\left(1+x^{2}+y^{2}+a^{2}\left(x^{2}+y^{2}\right)^{2}\right)^{2}-4 x^{2} y^{2}}
\end{aligned}
$$

and in polar coordinates $(r, \varphi)$ with

$$
\begin{gathered}
x=r \cos \varphi, y=r \sin \varphi, 0<r<\infty, 0 \leq \varphi<2 \pi \\
K^{\prime}(x, y)=\frac{1}{\pi} \cdot \frac{r^{2} \sin 2 \varphi}{\left(1+r^{2}+a^{2} r^{4}\right)^{2}-r^{4}(\sin 2 \varphi)^{2}} .
\end{gathered}
$$

We want to get estimate for $E^{o}(a)$ from below by choosing a test function

$$
f_{*}(x)=x \exp \left(-\frac{1}{2} h^{2} x^{2}\right),
$$

$h>0$ to be specified later, $h H=1$, and doing explicit calculations of quadratic forms. So

$$
E^{o}(a) \geq \frac{\left\langle K^{o} f_{*}, f_{*}\right\rangle}{\left\langle f_{*}, f_{*}\right\rangle}=1-\alpha(a) .
$$

Proposition 2. For a kernel (30), (32) with notations (33), (34) for small enough $a>0$

$$
\alpha(a) \leq C a^{2 / 5}
$$

$C$ an absolute constant, $C<3$.

Proof.

$$
\begin{aligned}
& \left\langle f_{*}, f_{*}\right\rangle=\int_{-\infty}^{\infty} x^{2} \exp \left(-h^{2} x^{2}\right) d x=H^{3} \int_{-\infty}^{\infty} \int_{-\infty}^{\infty}(h x)^{2} \exp \left(-(h x)^{2}\right) d(h x)= \\
& =2 H^{3} \int_{0}^{\infty} y^{2} e^{-y^{2}} d y=H^{3} \int_{0}^{\infty} u^{1 / 2} e^{-u} d u=H^{3} \Gamma(3 / 2)=\frac{\sqrt{\pi}}{2} H^{3} .
\end{aligned}
$$

Next, by (31), (32),

$$
f_{*}(x) f_{*}(y)=x y \exp \left(-\frac{1}{2} h^{2}\left(x^{2}+y^{2}\right)\right)=\frac{1}{2} r^{2} \exp \left(-\frac{1}{2} h^{2} r^{2}\right) \sin 2 \varphi,
$$


and

$$
\begin{aligned}
\left\langle K^{o} f_{*}, f_{*}\right\rangle=\left\langle K^{\prime} f_{*}, f_{*}\right\rangle & =\iint_{\mathbb{R}^{2}} K^{\prime}(x, y) f_{*}(x) f_{*}(y) d x d y= \\
& =\frac{1}{\pi} \int_{0}^{\infty} \int_{0}^{2 \pi} \frac{e^{-\frac{1}{2} h^{2} r^{2}} r \frac{1}{2} r^{4}(\sin 2 \varphi)^{2} d \varphi d r}{C^{2}-A^{2}(\sin 2 \varphi)^{2}}
\end{aligned}
$$

where

$$
\begin{gathered}
C=1+r^{2}+a^{2} r^{4}, \\
A=r^{2} ; C^{2}=A^{2}+B^{2},
\end{gathered}
$$

and

$$
\begin{gathered}
B=\sqrt{C^{2}-A^{2}}=\sqrt{(C-A)(C+A)}, \\
C-A=1+a^{2} r^{4}, C+A=1+2 r^{2}+a^{2} r^{4} .
\end{gathered}
$$

Again,

$$
\left\langle K^{\prime} f_{*}, f_{*}\right\rangle=\int_{0}^{\infty} r^{5} \exp \left(-\frac{1}{2} h^{2} r^{2}\right) I(r) d r
$$

where

$$
I(r)=\frac{1}{2 \pi} \int_{0}^{2 \pi} \frac{(\sin 2 \varphi)^{2}}{C^{2}-A^{2}(\sin 2 \varphi)^{2}} d \varphi .
$$

We are lucky to have an explicit integration after observation that

$$
\int_{0}^{2 \pi} \cdots=8 \int_{0}^{\pi / 4} \cdots
$$

and substitution $t=\tan 2 \varphi$. Then

$$
\begin{aligned}
& (\sin 2 \varphi)^{2}=\frac{t^{2}}{1+t^{2}}, \quad(\cos 2 \varphi)^{2}=\frac{1}{1+t^{2}} \\
& d t=\frac{2 d \varphi}{(\cos 2 \varphi)^{2}} \quad \text { and } \quad d \varphi=\frac{1}{2} \cdot \frac{d t}{1+t^{2}}
\end{aligned}
$$

and

(48) $I(r)=\frac{1}{2 \pi} \cdot 8 \cdot \frac{1}{2} \int_{0}^{\infty}\left(-\frac{1}{1+t^{2}}\right) \frac{d t}{C^{2}\left(1+t^{2}\right)-A^{2} t^{2}}=$ 


$$
=\frac{2}{\pi} \int_{0}^{\infty}\left(1-\frac{1}{1+t^{2}}\right) \frac{d t}{C^{2}+B^{2} t^{2}} .
$$

By the elementary calculus

$$
\int_{0}^{\infty} \frac{d t}{p^{2}+q^{2} t^{2}}=\frac{\pi}{2} \cdot \frac{1}{p q}, \text { any } p, q>0
$$

and

$$
\frac{1}{1+t^{2}} \cdot \frac{1}{C^{2}+B^{2} t^{2}}=\left(\frac{1}{1+t^{2}}-\frac{B^{2}}{C^{2}+B^{2} t^{2}}\right) \cdot \frac{1}{A^{2}}
$$

Therefore, by (48)-(

$$
I(r)=\frac{1}{C B}-\frac{1}{A^{2}}\left(1-\frac{B^{2}}{C B}\right)=\frac{1}{A^{2}}\left[\frac{C}{B}-1\right] \equiv I_{1}(r)-I_{2}(r) .
$$

According to (43) put

$$
J_{\delta}=\int_{0}^{\infty} r^{5} \exp \left(-\frac{1}{2} h^{2} r^{2}\right) I_{\delta}(r) d r, \delta=1,2 .
$$

Then an easy part gives with $A^{2}=r^{4}$

$J_{2}=\int_{0}^{\infty} r \exp \left(-\frac{1}{2} h^{2} r^{2}\right) d r=H^{2} \int_{0}^{\infty} \exp \left(-\frac{1}{2} h^{2} r^{2}\right) d\left(\frac{1}{2} h^{2} r^{2}\right)=H^{2}$.

But with (41), (42)

$$
J_{1}=\int_{0}^{\infty} r \exp \left(-\frac{1}{2} h^{2} r^{2}\right) \frac{\left(1+r^{2}+a^{2} r^{4}\right) d r}{\sqrt{1+a^{2} r^{4}} \cdot \sqrt{1+2 r^{2}+a^{2} r^{4}}}
$$

and with

$$
\begin{gathered}
R=2 r^{2}, r d r=\frac{1}{4} d R \\
J_{1}=\int_{0}^{\infty} \exp \left(-\frac{1}{4} h^{2} R\right) \frac{\left(1+\frac{1}{2} R+\frac{1}{4} a^{2} R^{2}\right) \frac{1}{4} d R}{\left(1+\frac{1}{4} a^{2} R^{2}\right)^{1 / 2}\left(1+R+\frac{1}{4} a^{2} R^{2}\right)^{1 / 2}} .
\end{gathered}
$$

Finally, put

$$
w=\frac{1}{4} h^{2} R, R=4 H^{2} w
$$

So

$$
J_{1}=\int_{0}^{\infty} e^{-w} \frac{\left(1+2 H^{2} w+4 a^{2} H^{4} w^{2}\right) H^{2} d w}{\left(1+4 a^{2} H^{4} w^{2}\right)^{1 / 2}\left[1+4 H^{2} w+4 a^{2} H^{4} w^{2}\right]^{1 / 2}} .
$$

By (53) and (36), (33) — an easy part -

$$
\frac{J_{2}}{\left\|f_{*}\right\|^{2}}=\frac{H^{2}}{\sqrt{\pi} / 2 H^{3}}=\frac{2}{\sqrt{\pi}} h,
$$


but

$$
\begin{aligned}
& \text { (60) } \widetilde{J}_{1}=\frac{J_{1}}{\left\|f_{*}\right\|^{2}}= \\
& =\frac{2}{\sqrt{\pi}} \int_{0}^{\infty} e^{-w} \frac{H^{2} \cdot 2 H^{2}\left(\frac{1}{2} h^{2}+w+2(a H)^{2} w^{2}\right) d w}{H^{3} \cdot 2 H\left(\frac{1}{4} h^{2}+w+(a H)^{2} w^{2}\right)^{1 / 2}\left(1+4\left(a H^{2}\right)^{2}+w^{2}\right)^{1 / 2}}= \\
& =\frac{2}{\sqrt{\pi}} \int_{0}^{\infty} e^{-w}\left(1+4\left(a H^{2}\right)^{2} w^{2}\right)^{-1 / 2} \cdot \frac{w+\frac{1}{2} h^{2}+2(a H)^{2} w^{2}}{\left(w+\frac{1}{4} h^{2}+(a H)^{2} w^{2}\right)^{1 / 2}} d w .
\end{aligned}
$$

Put

$$
u=4 a^{2} H^{4} w^{2}
$$

and

$$
s=\frac{1}{4} h^{2}+(a H)^{2} w^{2}
$$

Then

$$
1-(1+u)^{-1 / 2}=\frac{u}{1+u+\sqrt{1+u}}=\frac{u}{2} \cdot g_{a}(w)
$$

and

$$
\begin{gathered}
0 \leq g_{a}(w)<1, \\
g(u) \rightarrow 1 \text { if } u \rightarrow 0 ; \rightarrow 0 \text { if } u \rightarrow \infty .
\end{gathered}
$$

For the second factor in the integrant on the right side of (60) we use elementary identities

$$
\begin{aligned}
\frac{w+2 s}{\sqrt{w+s}}-w^{1 / 2} & =\frac{w+2 s-(w(w+s))^{1 / 2}}{\sqrt{w+2 s}}= \\
& =\frac{s(3 w+4 s)}{\left(\sqrt{w+s}(w+2 s+w(w+s))^{1 / 2}\right)} \equiv s \cdot G(w)
\end{aligned}
$$

where

$$
G(w) \leq \frac{4(w+s)}{(w+s)[\sqrt{w+2 s}+\sqrt{w}]} \leq 2 w^{-1 / 2}
$$

and

$$
\begin{aligned}
& G(w) \rightarrow 2 w^{-1 / 2} \text { if } h \rightarrow 0, a H \rightarrow 0, \\
& \text { or } \frac{1}{2} w^{1 / 2} G(w) \rightarrow 1, \text { if } h \rightarrow 0, a H \rightarrow 0 .
\end{aligned}
$$

After these notations and observations we can continue to evaluate (60) and write

$$
\widetilde{J}_{1}=\frac{J_{1}}{\left\|f_{*}\right\|^{2}}=\frac{2}{\sqrt{\pi}} \int_{0}^{\infty} e^{-w}\left(1-\frac{1}{2} u g(w)\right)\left[w^{1 / 2}+s G(w)\right] d w=
$$




$$
\frac{2}{\sqrt{\pi}} \int_{0}^{\infty} e^{-w}\left[w^{1 / 2}-\frac{1}{2} u w^{1 / 2} g(w)+s G(w)-\frac{1}{2} u s g(w) G(w)\right] d w .
$$

Put correspondingly

$$
\widetilde{J}_{1}=F_{0}-F_{1}+F_{2}-F_{3}
$$

and let us do calculations and estimates under the assumption

$$
a \rightarrow 0, h \rightarrow 0, a H^{2} \rightarrow 0, h H=1 .
$$

Then

$$
\begin{gathered}
F_{0}=\frac{2}{\sqrt{\pi}} \int_{0}^{\infty} w^{1 / 2} e^{-w} d w=\frac{2}{\sqrt{\pi}} \Gamma\left(\frac{3}{2}\right)=1 \\
F_{1}=\frac{2}{\sqrt{\pi}} \int_{0}^{\infty} e^{-w} \frac{1}{2}\left(4 a^{2} H^{4} w^{2}\right) w^{1 / 2} g_{a}(w) d w= \\
=\frac{4}{\sqrt{\pi}} a^{2} H^{4} \int_{0}^{\infty} e^{-w} w^{5 / 2} g_{a}(w) d w=\frac{15}{2} a^{2} H^{4}\left(1+\varepsilon_{1}(a)\right)
\end{gathered}
$$

where $\varepsilon_{1}(a) \rightarrow 0$ under conditions (71) by (61)-(64) and Lebesgue dominated convergence theorem (LDCT). We use also that

$$
\Gamma\left(\frac{7}{2}\right)=\frac{5}{2} \cdot \frac{3}{2} \cdot \frac{1}{2} \cdot \Gamma\left(\frac{1}{2}\right)=\frac{15}{8} \sqrt{\pi}
$$

Next step is an evaluation of $F_{2}$. By (62) and (666), (67), (68)

$$
\begin{gathered}
F_{2}=\frac{2}{\sqrt{\pi}} \int_{0}^{\infty} e^{-w} s G(w) d w= \\
=\frac{2}{\sqrt{\pi}} \int_{0}^{\infty} e^{-w}\left[\frac{1}{4} h^{2}+(a H)^{2} w^{2}\right] G(w) d w= \\
=\frac{2}{\sqrt{\pi}} \cdot \frac{1}{4} h^{2} \int_{0}^{\infty} e^{-w} G(w) d w+\frac{2}{\sqrt{\pi}}(a H)^{2} \int_{0}^{\infty} e^{-w} w^{2} G(w) d w= \\
=\frac{1}{2 \sqrt{\pi}} h^{2} \Gamma\left(\frac{1}{2}\right)\left(1+\varepsilon_{2}(a)\right)+\frac{2}{\sqrt{\pi}}(a H)^{2} 2 \Gamma\left(\frac{5}{2}\right)\left(1+\varepsilon_{2}^{\prime}\right)= \\
=h^{2}+3(a H)^{2}+h^{2} \varepsilon_{2}(a)+(a H)^{2} \cdot 3 \varepsilon_{2}^{\prime}(a)
\end{gathered}
$$

where $\varepsilon_{2}(a) \rightarrow 0, \varepsilon_{2}^{\prime}(a) \rightarrow 0$ if (71) holds. One more step evaluates $F_{3}$.

$$
\begin{aligned}
& F_{3}=\frac{2}{\sqrt{\pi}} \int_{0}^{\infty} \frac{1}{2} e^{-w} u s g(w) G(w) d w= \\
= & \frac{1}{\sqrt{\pi}} \int_{0}^{\infty} e^{-w}\left(4 a^{2} H^{4} w^{2}\right)\left(\frac{1}{4} h^{2}+(a H)^{2} w^{2}\right) \cdot g(w) G(w) d w=
\end{aligned}
$$




$$
=\frac{1}{\sqrt{\pi}} \int_{0}^{\infty} e^{-w}\left[a^{2} H^{2} w^{2}+4 a^{4} H^{6} w^{4}\right] 2 w^{-1 / 2} \gamma(w) d w
$$

where $0<\gamma(w)<1, \gamma_{a}(w) \rightarrow 1$ if (71) holds. Therefore, by LDCT

$$
\begin{aligned}
F_{3}=\frac{2}{\sqrt{\pi}} a^{2} H^{2} \Gamma\left(\frac{5}{2}\right)\left(1+\varepsilon_{2}(a)\right) & +\frac{8}{\sqrt{\pi}} a^{4} H^{6} \Gamma\left(\frac{9}{2}\right)\left(1+\varepsilon_{3}^{\prime}(a)\right)= \\
= & {\left[\frac{3}{2} a^{2} H^{2}+\frac{105}{2} a^{4} H^{6}\right]\left(1+\varepsilon_{3}^{\prime \prime}(a)\right) }
\end{aligned}
$$

where $\varepsilon_{3}(a), \varepsilon_{3}^{\prime}, \varepsilon_{3}^{\prime \prime} \rightarrow 0$ if (71) holds.

Now we need to collect five terms $F_{j}, 0 \leq j \leq 3$, and $J_{2}$. Remind orders of these terms

$$
\begin{aligned}
F_{0} & =1 \text { by }(72) \\
F_{1} & \sim a^{2} H^{4} \text { by }(73) \\
F_{2} & \sim h^{2}+(a H)^{2} \text { by }(74) \\
F_{3} & \sim a^{2} H^{2}+a^{4} H^{6} \text { by (176) } \\
\widetilde{J}_{2} & \sim h \text { by }
\end{aligned}
$$

Under conditions (71) with

$$
a^{2} H^{2}=\left(a^{2} H^{4}\right) \cdot h^{2}, \quad a^{4} H^{6}=\left(a^{2} H^{4}\right)^{2} \cdot h^{2}
$$

if $F_{1} \sim \widetilde{J}_{2}$ two terms $F_{1}, \widetilde{J}_{2}$ majorize $F_{2}, F_{3}$, i.e.,

$$
\frac{\left(F_{2}+F_{3}\right)}{h}=O(h)
$$

and

$n(a)=\frac{\left\langle K^{\prime} f_{*}, f_{*}\right\rangle}{\left\langle f_{*}, f_{*}\right\rangle}=1-\widetilde{J}_{2}-F_{1}+O\left(h^{2}\right)=1-\frac{2}{\sqrt{\pi}} h-\frac{15}{2} a^{2} H^{4}+O\left(h^{2}\right)$

if

$$
h \sim a^{2} H^{4} \quad \text { or } \quad h=\lambda a^{2 / 5}
$$

SO

$$
n(a)=1-\left(\frac{2}{\sqrt{\pi}} \lambda+\frac{15}{2} \cdot \frac{1}{\lambda^{4}}\right) a^{2 / 5}+O\left(a^{4 / 5}\right) .
$$

We will not get sharp constants in the final result (see Theorem 8) but at least at this step we'll anyway choose the best possible $g_{*}$ by 
finding

$$
g_{*}=\min \left(\frac{2}{\sqrt{\pi}} \lambda+\frac{15}{2} \cdot \frac{1}{\lambda^{4}}\right) .
$$

It is attained for $\lambda_{*}$,

$$
\lambda_{*}=(15 \sqrt{\pi})^{1 / 5}
$$

SO

$$
g_{*}=\frac{1}{2}\left(5^{6} \cdot 3 \cdot \pi^{-2}\right)^{1 / 5} \approx 2.718305798 \ldots<3 .
$$

Now, in (33) we are ready to choose

$$
h=\lambda_{*} a^{2 / 5} \text {. }
$$

Therefore, we've shown, by (81) and (84), that

$$
\frac{\left\langle K f_{*}, f_{*}\right\rangle}{\left\langle f_{*}, f_{*}\right\rangle}=1-g_{*} a^{2 / 5}+O\left(a^{4 / 5}\right) \geq 1-3 a^{2 / 5}
$$

for a small enough $a$. We proved (35) and Proposition 2.

3. As we noticed in (21)-(24)

$$
E^{o}(a)=\left\|K_{a 1}^{o}\right\| \text {. }
$$

To evaluate this quantity from above, we'll use the following Schur lemma [Sc].

Lemma 3. Let $A$ be an integral symmetric operator, i.e., $A(x, y)=$ $A(y, x)$,

in $L^{2}\left(\mathbb{R}^{m}\right)$. Then

$$
A: f \rightarrow \int_{\mathbb{R}^{m}} A(x, y) f(y) d \mu
$$

$$
\|A\| \leq \sup _{x} \int_{\mathbb{R}^{m}}|A(x, y)| d \mu(y) .
$$

(See more about Schur lemma, or Schur test, in [DK], Section 3, or [HS], Theorem 5.2. More general statements in the context of the operator interpolation theory can be found in [Mi, Ca.)

It is quite surprising that this lemma gives us the sharp up to the second term estimate of the norm $\left\|K^{o}\right\|$.

Proposition 4. Let $K^{\prime}$ be a kernel (30). Then

$$
\sup _{x} \int_{-\infty}^{\infty}\left|K^{\prime}(x, y)\right| d y \leq 1-\beta(a)
$$

where

$$
\beta(a) \geq c a^{2 / 5} \text { for small enough } a>0
$$


$c$ being an absolute constant, $c>2 / 3$.

Proof is straightforward.

$$
K^{\prime}(x, y)=-K(-x, y)
$$

so we can consider only $x>0$ in (88).

Then

$$
\begin{aligned}
& J \equiv \int_{\mathbb{R}}\left|K^{\prime}(x, y)\right| d y= \\
& \quad=\frac{2 x}{\pi} \cdot 2 \int_{0}^{\infty} \frac{y d y}{\left(1+x^{2}+y^{2}+a^{2}\left(x^{2}+y^{2}\right)^{2}\right)^{2}-4 x^{2} y^{2}}
\end{aligned}
$$

and after substitution $Y=y^{2}$

$$
J=\frac{2 x}{\pi} \int_{0}^{\infty} \frac{d Y}{\left(1+x^{2}+Y+a^{2}\left(x^{2}+Y\right)^{2}\right)^{2}-4 x^{2} Y} .
$$

Notice that the denominator

$$
\begin{gathered}
D=a^{4}\left(x^{2}+Y\right)^{4}+2\left(1+x^{2}+Y\right)\left(x^{2}+Y\right)^{2} a^{2}+1+2\left(x^{2}+Y\right)+\left(Y-x^{2}\right)^{2}> \\
>2 a^{2} x^{6}+\left(Y-x^{2}\right)^{2}+2\left(Y-x^{2}\right)+1+4 x^{2}=\left(Y-x^{2}+1\right)^{2}+4 x^{2}+2 a^{2} x^{6} .
\end{gathered}
$$

Therefore, with $\xi=Y-x^{2}+1$, and $\xi=2 x t$,

$$
\begin{aligned}
& J<\frac{2 x}{\pi} \int_{-x^{2}+1}^{\infty} \frac{d \xi}{\xi^{2}+4 x^{2}+2 a^{2} x^{6}}< \\
&<\frac{2 x}{\pi} \int_{-x / 2}^{\infty} \frac{2 x d t}{4 x^{2}\left[t^{2}+1+\frac{1}{2} a^{2} x^{4}\right]} \equiv p(x) .
\end{aligned}
$$

By (49)

$$
p(x)=\left(1+\frac{1}{2} a^{2} x^{4}\right)^{-1 / 2}\left[\frac{1}{2}+\frac{1}{\pi} \arctan \frac{x}{2 \sqrt{1+\frac{1}{2} a^{2} x^{4}}}\right] .
$$

In (95)) we have two positive factors, each of them less than 1 , so if we expect their product to be close to 1 we want each of them to be close to 1 . It will be achieved if

$$
a^{2} x_{*}^{4} \rightarrow 0, x_{*} \rightarrow \infty
$$

when $a \rightarrow 0$ where

$$
x_{*}=x_{*}(a), p\left(x_{*}\right)=\max _{x>0} p(x) .
$$


So far we used rough inequalities (93)-(94) and we do not expect to get sharp constants. Therefore, we do not look for finding exact $x_{*}$ but we want reasonable estimates for

$$
p^{*}=p\left(x_{*}\right)=\max _{x>0} p(x) .
$$

Notice that for $v>1$

$$
\text { (99) } \begin{aligned}
& \frac{1}{\pi} \arctan v=\frac{1}{2}-\frac{1}{\pi} \int_{v}^{\infty} \frac{d y}{1+y^{2}} \leq \\
& \leq \frac{1}{2}-\frac{1}{\pi} \frac{v^{2}}{1+v^{2}} \int_{v}^{\infty} \frac{d y}{y^{2}}<\frac{1}{2}-\frac{1}{\pi} \cdot \frac{1}{v}\left(1-\frac{1}{v^{2}}\right) \\
& \quad<\frac{1}{2}-\frac{7}{22} \cdot \frac{1}{v} \text { if } v>30 .
\end{aligned}
$$

Therefore, by (94), (98), (99)

$$
p(x) \leq\left(1+\frac{1}{2} a^{2} x^{4}\right)^{-1 / 2}\left(1-\frac{7}{11} \cdot \frac{1}{x}\right)
$$

and by elementary inequality

$$
(1+w)^{-1 / 2}<1-\frac{9}{20} w \quad \text { if } 0<w<\frac{7}{50}
$$

so with $x>1$

$$
\begin{aligned}
p(x)< & \left(1-\frac{9}{40} a^{2} x^{4}\right)\left(1-\frac{7}{11} \cdot \frac{1}{x}\right)= \\
=1-\left(\frac{9}{40} a^{2} x^{4}+\frac{7}{11} \cdot \frac{1}{x}\right) & +\frac{63}{440} \cdot a^{2} x^{3}< \\
& <1-\left(\frac{9}{110} a^{2} x^{4}+\frac{7}{11} \cdot \frac{1}{x}\right) .
\end{aligned}
$$

Again, as in (82)-(85) we look for

$$
g^{*}=\min _{\mu>0}\left(\frac{7}{11} \cdot \frac{1}{\mu}+\frac{9}{110} \mu^{4}\right)
$$

and

$$
\mu^{*}=\left(\frac{7}{11} \cdot \frac{110}{4 \cdot 9}\right)^{1 / 5}=\left(\frac{35}{18}\right)^{1 / 5} \approx 1.142244585 .
$$

The choice

$$
x^{*}=\mu^{*} a^{-2 / 5}
$$


gives the best result in the right side of (102). This leads us to the inequality

$$
p(x)<1-g^{*} a^{2 / 5}
$$

where by (103) $g^{*}=\frac{35}{44}\left(\frac{18}{35}\right)^{1 / 5} \approx 0.696395987$

$$
g^{*}>\frac{2}{3} .
$$

We proved (8ㅛ)-(89) with $c=\frac{2}{3}$.

4. In (33) we've chosen a smooth cut-off but calculations of Sect. 2 could be done (as long as we do not try to find sharp constants) with other $f_{*}$ 's, say,

$$
f_{*}(x)= \begin{cases}x, & |x| \leq H \\ 0, & |x|>H\end{cases}
$$

Then

$$
\left\langle f_{*}, f_{*}\right\rangle=\frac{2}{3} H^{3} .
$$

Again, the integral (43) will play important role, i.e., we use the following.

Lemma 5. If $C>A>0$ then

$$
I=\frac{1}{2 \pi} \int_{0}^{2 \pi} \frac{(\sin 2 \varphi)^{2} d \varphi}{C^{2}-A^{2}(\sin 2 \varphi)^{2}}=\frac{1}{A^{2}}\left[\frac{C}{B}-1\right]
$$

where

$$
A^{2}+B^{2}=C^{2}, B>0 .
$$

Proof was given in Sect 2, formulas (44)-(51). Now

$$
n:=\left\langle K^{\prime} f_{*}, f_{*}\right\rangle=\iint_{|x|,|y| \leq H} K^{\prime}(x, y) x y d x d y
$$

and with the integrand being positive we have

$$
\int_{0}^{H}\left[\frac{C}{B}-1\right] r d r \leq n \leq \int_{0}^{H \sqrt{2}}\left[\frac{C}{B}-1\right] r d r .
$$

The same analysis as on pp. 7 10 will shows that if

$$
H^{-1}=h=\lambda a^{2 / 5}
$$

then

$$
\frac{n}{2 / 3 H^{3}}=1-\varphi(a)
$$


where

$$
\varphi(a) \leq C_{4} a^{2 / 5}
$$

although because of (113) with different upper bounds of integration this absolute constant $C_{4}$ will be worse than in (84) or (86), even if we will try to choose $\lambda$ appropriately.

5. In previous section we saw that Schur lemma gives good upper estimates (88)-(89) of the norm of an integral operator with the kernel $K^{\prime} \in(30)$, (32). But an attempt to apply Lemma 3 to the kernel $K \in(2), T=1$, does not give a right order of the term which is an analogue of $\beta$ in (88). Even if we take $x=0$

$$
\begin{aligned}
& \int_{\mathbb{R}} K(0, y) d y=\frac{2}{\pi} \int_{0}^{\infty} \frac{d y}{1+y^{2}+a^{2} y^{4}}= \\
&=1-\frac{2}{\pi} a^{2} \int_{0}^{\infty} \frac{y^{4} d y}{\left(1+y^{2}\right)\left(1+y^{2}+a^{2} y^{4}\right)}= \\
&=1-C(a) \cdot a \text { with } C(a) \rightarrow 1(a \rightarrow 0) .
\end{aligned}
$$

So even if the estimate

$$
\left\|K_{a}\right\| \leq 1-\frac{3}{4} a, \quad a \leq a^{*} \text { for some } \quad a^{*}>0
$$

were correct it would be far away from the below estimate (86).

However, a more skillful use of Schur lemma (or its proof) combined with Uncertainty Principle (in its additive form) gives (!) good estimates of the norm $\left\|K_{a}\right\|$ of the full operator (11), (2). These constructions have been suggested by Fedor Nazarov [private communication, Oct. 26, 2006].

Lemma 6 (Uncertainty Principle). Let $f \in L^{2}\left(\mathbb{R}^{1}\right), \int_{\mathbb{R}^{1}}|f(x)|^{2} d x=1$.

For any $h>0, h H=1$, one of two inequalities (a) or (b) holds:

(a) $\int_{|x| \geq H}|f(x)|^{2} d x \geq \frac{1}{9}$, or

(b) $\int_{|s| \geq h}|\tilde{f}(s)|^{2} d s \geq \frac{1}{9}$,

where

$$
\widetilde{f}(s)=\mathcal{F} f \equiv \frac{1}{\sqrt{2 \pi}} \int_{-\infty}^{\infty} e^{-i s x} f(x) d x
$$

i.e., $\mathcal{F}$ is standard unitary Fourier operator in $L^{2}$.

This is a version of the celebrated Landau-Pollack-Slepian inequalities (Additive Uncertainty Principle). In Appendix we'll discuss it and 
give a proof of Lemma 6 to make the present paper a self-contained exposition.

Now we will give an estimate from above of the norm of $K$-image, $K \in(1),(2), T=1$,

$$
K f(x)=\int_{-\infty}^{\infty} K(x, y) f(y) d y .
$$

We can assume $[$ see (18) $-(25)]$ that $f(x) \geq 0$ if

$$
\|K\|=\|K f\|,\|f\|=1 \text {. }
$$

If in Lemma 6 (b) holds we do the following estimates:

$$
K f(x) \leq K_{0} f(x)=\frac{1}{\pi} \int_{-\infty}^{\infty} \frac{f(y) d y}{1+(x-y)^{2}}=k * f,
$$

and $\mathcal{F}$ being isometry

$$
\begin{aligned}
\|K f(x)\|_{2}^{2} \leq\|k * f\|_{2}^{2} & =\|\widetilde{k}(s) \cdot \widetilde{f}(s)\|_{2}^{2}= \\
=\int_{-\infty}^{\infty} e^{-2|s|}|\widetilde{f}(s)|^{2} d s & =1-\int_{-\infty}^{\infty}\left(1-e^{-2|s|}\right)|\widetilde{f}(s)|^{2} d s \leq \\
\leq & 1-\left(1-e^{-2 h}\right) \cdot \frac{1}{9}<1-\frac{1}{6} h, \text { if } h \leq \frac{1}{4} .
\end{aligned}
$$

We used (b) and elementary inequalities

$$
1-e^{-v} \geq \frac{3}{4} v
$$

if

$$
0<v<\frac{1}{2}
$$

If (a) holds we do as Schur did, i.e., by Cauchy inequality, with $K \cdot f=$ $K^{1 / 2}\left(K^{1 / 2} f\right)$,

$$
(K f(x))^{2} \leq \int K(x, \xi) d \xi \cdot \int K(x, y) f^{2}(y) d y \leq \int K(x, y) f^{2}(y) d y
$$

and

where

$$
\int(K f(x))^{2} d x \leq \int M(y) f^{2}(y) d y
$$

$$
M(y)=\int_{-\infty}^{\infty} \frac{1}{\pi} \cdot \frac{d x}{1+(x-y)^{2}+a^{2}\left(x^{2}+y^{2}\right)^{2}} .
$$

For any $y M(y) \leq 1$ but if $|y| \geq H$ we get a better estimate: notice that if $|x-y| \leq 1$ then

$$
1+(x-y)^{2}+a^{2}\left(x^{2}+y^{2}\right)^{2} \geq\left[1+(x-y)^{2}\right]\left(1+\frac{a^{2}}{2} y^{4}\right)
$$


SO

$$
\begin{aligned}
M(y) & \leq \int_{|x-y| \leq 1} \frac{1}{\pi} \frac{d x}{\left(1+(x-y)^{2}\right)\left(1+\frac{a^{2}}{2} y^{4}\right)}+\int_{|x-y| \geq 1} \frac{1}{\pi} \frac{d x}{1+(x-y)^{2}} \leq \\
& \leq \frac{1}{2} \cdot \frac{1}{1+\frac{1}{2} a^{2} H^{4}}+\frac{1}{2}=1-\frac{1}{2}\left(1-\frac{1}{1+\frac{1}{2} a^{2} H^{4}}\right)<1-\frac{1}{6} a^{2} H^{4}
\end{aligned}
$$

if $a^{2} H^{4}<1$. Let us choose such $h>0$ that

$$
\frac{1}{6} h=\frac{1}{6} a^{2} H^{4}, h H=1,
$$

i.e.,

$$
h=\left(a^{2}\right)^{1 / 5} .
$$

Then inequalities (114) and (115) give the same estimate for the case (b) and (a), correspondingly. By Lemma 6, it covers all possible cases. These inequalities give the upper bound of the square of the norm $\|K\|^{2}$; therefore

$$
\|K\| \leq\left(1-\frac{1}{6} h\right)^{1 / 2}<1-\frac{1}{12} a^{2 / 5} .
$$

Hence, we proved the following

Proposition 7. Let $K_{a}$ be an integral operator in $L^{2}\left(\mathbb{R}^{1}\right)$ with a kernel

$$
K_{a}(x, y)=\frac{1}{\pi} \cdot \frac{1}{1+(x-y)^{2}+a^{2}\left(x^{2}+y^{2}\right)^{2}} .
$$

Then

$$
\left\|K_{a}\right\|=E(u)=1-\gamma(a)
$$

where $\gamma(a) \geq \frac{1}{12} a^{2 / 5}$ for small enough $a>0$.

Prop 2 gives estimates from above of the deficiency term $\psi$ in (9) in the case of the subspace of odd functions. But with inequalities (12) of Lemma 1, Prop 7 and 2 together complete the proof of the following statement.

Theorem 8. Let $E(a)=E^{e}(a)$ and $E^{o}(a)$ be the largest eigenvalues of the integral operator $K_{a} \in(116)$ on subspaces of even and odd functions correspondingly. Then for $a>0$ small enough

$$
3 a^{2 / 5} \geq 1-E^{o}(a)>1-E^{e}(a)=1-E(a) \geq \frac{1}{12} a^{2 / 5} .
$$


6. Now we can give an asymptotic of $\tau(a)$ in the solution (10) of the equation $E^{o}(a, T)=1$.

Lemma 9. The norm $N(a, T)$ of an operator $K_{a T} \in(1)$ has the property

$$
N(a b, T / b)=b N(a, T), \forall T, a, b>0 .
$$

The same is true for the norms $N^{e}, N^{o}$ of $K_{a T}^{e}, K_{a T}^{o}$, restrictions of $K \in$ (1) to the subspaces of even and odd functions.

Proof. Notice that the kernel $K \in$ (11), with $x=b \xi, y=b \eta$, becomes

$$
\begin{aligned}
& K(b \xi, b \xi)=\frac{1}{\pi} \cdot \frac{1}{T^{2}+b^{2}(\xi-\eta)^{2}+a^{2} b^{4}\left(\xi^{2}+\eta^{2}\right)^{2}}= \\
& =\frac{1}{b^{2}} \cdot \frac{1}{\pi} \cdot \frac{1}{(T / b)^{2}+(\xi-\eta)^{2}+(a b)^{2}\left(\xi^{2}+\eta^{2}\right)^{2}} .
\end{aligned}
$$

However,

$$
\begin{aligned}
& \sup _{\|\varphi\|=1} \iint K(b \xi, b \eta) \varphi(\xi) \varphi(\eta) d \xi d \eta= \\
& \quad=\sup _{\|\varphi\|=1} \frac{1}{b} \iint K(x, y) \frac{\varphi(x / b)}{\sqrt{b}} \cdot \varphi(y / b) \sqrt{b} d x d y=\frac{1}{b} N(a, T),
\end{aligned}
$$

because $\|\psi\|=1$, where $\psi(x)=\frac{1}{\sqrt{b}} \varphi(x / b)$. It implies

$$
\frac{1}{b^{2}} N(a b, T / b)=\frac{1}{b} N(a, T)
$$

i.e., (117) holds.

If we take in (118) only odd $\varphi$ we come to an identity

$$
N^{o}(a b, T / b)=b N^{o}(a, T)
$$

as well.

The same comment leads to such an identity for $N^{e}$ although we do not need to say this because $N^{e} \equiv N$ anyway.

Put $b=T$ in (120); then we have

$$
N^{o}(a T ; 1)=T N^{o}(a, T),
$$

and the equation $E^{o}(a, T)=1$, or - the same $-N^{o}(a, T)=1$, becomes an equation

$$
N^{o}(a T ; 1)=T \text {. }
$$

By Prop 2 and 4

$$
N^{o}(t ; 1)=1-\psi(t)
$$


and

$$
c_{1} t^{2 / 5} \leq \psi(t) \leq c_{2} t^{2 / 5}
$$

for small $t$. If $T=1-\tau(a)$ and $\tau(a) \rightarrow 0(a \rightarrow 0)$ as in (10), the equation (122) links $\tau$ and $\psi$ :

$$
1-\psi(a T)=T
$$

and

$$
\psi(a(1-\tau(a))=\tau(a)
$$

With $\tau(a) \rightarrow 0$

$$
\frac{1}{2}<1-\tau(a)<1
$$

so (126) and (124) imply

$$
\begin{aligned}
& C_{1}\left(\frac{1}{2} a\right)^{2 / 5} \leq C_{1}[a(1-\tau(a))]^{2 / 5} \leq \psi(a(1-\tau(a))) \equiv \tau(a) \leq \\
& \leq C_{2}[a(1-\tau(a))]^{2 / 5} \leq C_{2} a^{2 / 5}
\end{aligned}
$$

We proved the following.

Proposition 10. The temperature shift $\tau(a) \in(10)$ has estimates

$$
c a^{2 / 5} \leq \tau(a) \leq C a^{2 / 5}
$$

for small enough $a>0$, where $c, C$ are absolute constants.

Remark. If we would know that $\lim \psi(a) a^{-2 / 5}$ existed and were equal to $L$, then the same argument would tell us that

$$
\lim _{a \rightarrow 0} \tau(a) \cdot a^{-2 / 5}=L .
$$

7. Comments and questions.

7.1. Proposition 10 and Theorem 8 give two-side estimates for $\varphi(a)$, $\psi(a)$ and $\tau(a)$ - see (8) $-(10)$, but no information about existence of the limits $L^{e}$ or $L^{o}=L$ or their numerical values. (We explained this would-be equality in Remark above.)

But a natural conjecture would go far beyond these limits.

Let $\left\{E_{j}(a)\right\}, E_{0}(a) \geq E_{1}(a) \geq \ldots$, be a sequence of eigenvalues of an operator $K_{a}$. For any $j=0,1, \ldots E_{j}(a)=1-\varphi_{j}(a)$, and - we would conjecture -

$$
\lim \varphi_{j}(a) a^{-2 / 5}=\mu_{j} \text { exists, }
$$

and $\left\{\mu_{j}\right\}$ are eigenvalues of a (somewhere hidden) pseudo-differential operator $M$. 
This conjecture is inspired by H. Widom's analysis [Wi1, Wi2, Wi3] of integral operators

$$
T_{a}: f \rightarrow \int_{-1}^{1} \frac{1}{a} \rho\left(\frac{x-y}{a}\right) f(y) d y
$$

in $L^{2}(I), I=[-1 ;+1],[\mathrm{KMS}, \mathrm{Pa}]$. He assumes that

$$
R(s)=\int_{-\infty}^{\infty} e^{i x s} \rho(x) d x
$$

satisfies the following:

(i) $\lim R(s)=0$ when $s \rightarrow \pm \infty$.

(ii) $\max \{R(\xi)$ : $|\xi|>\delta\}<M$ for each $\delta>0$.

(iii) $\lim _{s \rightarrow 0}|s|^{-\alpha}(M-R(s))=c(0<c<\infty ; 0<\alpha<\infty)$.

Then a positive definite kernel $V(x, y)$ is given, and its eigenvalues $\lambda_{1} \geq \lambda_{2} \geq \ldots$ are linked with eigenvalues $\mu_{j}\left(T_{A}\right) \geq \mu_{j+1}\left(T_{A}\right)$ in the following way. For fixed $j$

$$
\lim _{a \rightarrow 0}\left(M-\mu_{j}\left(T_{A}\right)\right) a^{-\alpha}=c \lambda_{j}^{-1}
$$

moreover, each sequence of $A^{\prime}$ 's $(A a=1)$ tending to infinity has a subsequence for which $\psi_{j}(a), T_{A} \psi_{j}(a)=\mu_{j} \psi_{j}(a)$, converges in $L^{2}(I)$ to an eigenfunction of $V$ belonging to the eigenvalues $\lambda_{j}$. See details in Wi2].

Using Weyl symbols H. Widom gave (private communication) a heuristic argument which leads to a conjecture that this operator $M$ exists, it has a symbol $|s|+4 x^{4}$, or in other terms it is determined by the quadratic form

$$
<M f, f>=<|s| \tilde{f}(s), \tilde{f}(s)>+<4 x^{4} f(x), f(x)>.
$$

7.2. An integral operator (11)-(2) was brought to my attention by P. Krotkov and their analysis of models in superconductivity. From mathematical point of view, the kernel (2) is interesting because

- it is NOT translation invariant,

- a polynomal in the denominator is NOT homogenuous, it has terms of order 2 and 4 .

Although our analysis and results could be extended to a broader family of such kernels, the complete understanding of an interplay of orders of terms depending on $(x-y)$ and $(x+y)$, or $\left(x^{2}+y^{2}\right)$, would be very instructive.

Notice, for example, that the following is true. 
Proposition 11. Let

$$
K_{a}(x, y)=\frac{1}{\pi} \cdot \frac{1}{1+(x-y)^{2}+a^{2}\left(x^{2}+y^{2}\right)^{t}},
$$

$t>0$ fixed, $a>0$ goes to zero. Then its norms $N=N^{e}$ and $N^{o}$ satisfy inequalities

$$
c a^{2 /(1+2 t)} \leq 1-N^{e}(a)<1-N^{o}(a) \leq C a^{2 /(1+2 t)}, a \leq a^{*},
$$

where $c, C$ are constants depending on $t$ but not on $a$.

The operator $K_{a}$ with a kernel (129) is compact for any $t>0, a>0$.

The conjecture of Section 7.1 can easily be formulated for this example as well. How to prove it?

Acknowledgement. The author had interesting and useful discussions with F. Nazarov and H. Widom; he thanks them for valuable remarks and insights. The present paper has been completed in Fall 2007 during my stay at Weizmann Institute of Science, Rehovot, Israel, as Weston Visiting Professor. I thank Weizmann Institute for hospitality and stimulating environment.

Appendix

Additive Uncertainty Principle.

To make our paper self-contained we'll give a proof of Lemma 6. Some details follow closely to [FS], Sect. 8.

Let $P, R$ be two orthogonal projectors in a (real or complex) Hilbert space $H$. Put $E=\Im P, L=\Im R$.

Lemma 12. The norm of the product

$$
\|R P\|=b
$$

where

$$
b=\sup \{\langle u, v\rangle \mid P u=u, Q v=v,\|u\| \leq 1,\|v\| \leq 1\}
$$

and

$$
b^{2}=\|P R P\|
$$

Indeed,

$$
\|R P\|=\sup _{\|f\|,\|g\| \leq 1}\langle R P f, g\rangle
$$

but by (132)

$$
\langle R P f, g\rangle=\langle P f, R g\rangle=\|P f\| \cdot\|R g\| \cdot\left\langle u^{*}, v^{*}\right\rangle \leq b
$$


where $u^{*}=\frac{P f}{\|P f\|}, v^{*}=\frac{R g}{\|R g\|}$. On another side, for some sequence $\left\{u_{n}, v_{n}\right\} \subset E \times L,\left\|u_{n}\right\|,\left\|v_{n}\right\| \leq 1$,

$$
b=\lim \left\langle u_{n}, v_{n}\right\rangle=\lim \left\langle P u_{n}, R v_{n}\right\rangle \leq\|R P\| .
$$

Therefore (135) and (136) implies (131).

(133) just means that $\|A\|^{2}=\left\|A^{*} A\right\|$ where

$$
A=R P, \quad A^{*}=P R \quad \text { and } \quad A^{*} A=P R P .
$$

The geometric core of this Appendix is

Lemma 13. Let $u, v$ be unit vectors in $H$, and

$$
\langle u, v\rangle=T,|T|=t .
$$

Then for any $g \in H,\|g\|=1$.

$$
q^{2}:=|\langle g, u\rangle|^{2}+|\langle g, v\rangle|^{2} \leq 1+t .
$$

Proof. If $t=0$ this is Pythagor's identity. If $t=1$ this is Cauchy inequality.

In the case $0<t<1$ we can choose a subspace $K, \operatorname{dim} K=3$, $K \supset \operatorname{LinSpan}\{u, v, g\}$ and an o. n. b. $\left(e_{j}\right)_{1}^{3}$ in $K$ in such a way that

$$
\left\{\begin{array}{l}
u=(1,0,0) \\
v=(\bar{T}, \tau, 0), \tau=\sqrt{1-|T|^{2}} \geq 0 \\
g=(x, y, z) \in \mathbb{C}^{3},|x|^{2}+|y|^{2}+|z|^{2}=1 .
\end{array}\right.
$$

For any $h, 0<h=1 / H$ by Cauchy inequality

$$
\begin{aligned}
& q^{2}=|x|^{2}+|x T+y \tau|^{2}= \\
& =|x|^{2}\left(1+t^{2}\right)+|y|^{2} \tau^{2}+2 \Re(H x T)(\bar{y} \tau h) \leq \\
& \quad \leq|x|^{2}\left(1+t^{2}+H^{2} t^{2}\right)+|y|^{2}\left(1-t^{2}\right)\left(1+h^{2}\right)
\end{aligned}
$$

and the choice $h=\frac{t}{1-t}$ gives an inequality (139)

$$
q^{2} \leq\left(|x|^{2}+|y|^{2}\right)(1+t) \leq(1+t) .
$$

We are ready to prove the following

Proposition 14. For any $f,\|f\|=1$,

$$
r^{2}:=\|f-P f\|^{2}+\|f-R f\|^{2} \geq 1-b .
$$

Proof. $P$ is an orthogonal projector so $\langle P f, f-P f\rangle=0$; then

$$
\langle f, P f\rangle=\|P f\|^{2},\|P f\|=\langle f, u\rangle
$$

where $u=P f /\|P f\|$ and

$$
1=\|f\|^{2}=\|P f\|^{2}+\|f-P f\|^{2} .
$$


The same argument gives a vector

$$
v=R f /\|R f\|
$$

such that

$$
\|R f\|=\langle f, v\rangle
$$

and

$$
1=\|R f\|^{2}+\|f-R f\|^{2} .
$$

Therefore, (142) can be rewritten as

$$
1-|\langle f, u\rangle|^{2}+1-|\langle f, v\rangle|^{2} \geq 1-b
$$

or

$$
|\langle f, u\rangle|^{2}+|\langle f, v\rangle|^{2} \leq 1+b
$$

But

$$
|\langle u, v\rangle|=|\langle P u, R v\rangle|=|\langle R P u, v\rangle| \leq b
$$

by Lemma 12. Now (148) follows from (139) of Lemma 13.

Example 15. If $V$ is a unitary operator in $H$ then

$$
Q=V^{*} R V
$$

is an orthogonal projector as well:

$$
Q^{2}=V^{*} R V V^{*} R V=V^{*} R R V=V^{*} R V
$$

and

$$
Q^{*}=V^{*} R^{*} V=V^{*} R V=Q .
$$

The previous statements for $P, Q$ and unitarity of $V$ and $V^{*}$ give us

$$
b=\|Q P\|=\left\|V^{*} R V P\right\|=\|R V P\|
$$

and

$$
b^{2}=\left\|(R V P)^{*} R V P\right\|=\left\|P V^{*} R V P\right\|=\lambda_{0} .
$$

[If this block $T_{0}=P T P$ of an operator

$$
T=V^{*} R V
$$

is compact then

$$
b^{2}=\lambda_{0}, \lambda_{0}=\lambda_{0}(T)
$$

being its highest eigenvalue.]

Moreover, By Proposition [14, for any $f,\|f\|=1$,

$$
\|f-P f\|^{2}+\|f-Q f\|^{2} \geq 1-b .
$$

However

$$
\|f-Q f\|=\left\|f-V^{*} R V f\right\|=\|V f-R V f\|
$$


and the inequality (143) by (151) and (155) can be rewritten as:

$$
\|f-P f\|^{2}+\|(V f)-R(V f)\|^{2} \geq 1-b .
$$

We proved the following

Theorem 16. Let $P, R$ be orthogonal projectors in a (real or complex) Hilbert space $H$, and $V: H \rightarrow H$ a unitary operator. Then for any $f$, $\|f\|=1$

$$
\|f-P f\|^{2}+\|V f-R V f\|^{2} \geq 1-b
$$

where $1 \geq b \geq 0$,

$$
b^{2}=\left\|P V^{*} R V P\right\| .
$$

This inequality (159) is an Abstract Additive Uncertainty Principle.

Corollary 17. Under conditions of Theorem 16

$$
\max \left\{\|f-P f\|^{2} ;\|V f-R V f\|^{2}\right\} \geq \frac{1-b}{2} .
$$

Of course, the main example for us is $H=L^{2}(\mathbb{R})$ with a unitary operator $V=\mathcal{F}$, the Fourier transform

$$
\widetilde{f}(s)=\mathcal{F} f=\frac{1}{\sqrt{2 \pi}} \int_{-\infty}^{\infty} e^{-i s x} f(x) d x,
$$

and projectors $P=R$ where

$$
P f=f(x) \chi_{I}(x), \quad I=[-1,+1] .
$$

In this case

$$
T_{0}=P V^{*} R V P: f \rightarrow\left(\int_{-1}^{1}\left(\int_{-1}^{1} e^{-i s x} f(x) d x\right) e^{-i s y} d s\right) \chi(x)
$$

or

$$
\begin{array}{cl}
\left(T_{0} f\right)(y)=\frac{1}{\pi} \int_{-1}^{1} \frac{\sin (y-x)}{y-x} f(x) d x, & -1 \leq y \leq 1 \\
0, & \text { if }|y|>1
\end{array}
$$

Now remaining "hard analysis" question is to evaluate the norm $b^{2}=\lambda_{0}$ of this operator. The original paper $[\mathrm{SP}$ ] gives the value 0.57258 . We'll give a worse (larger) estimate. It comes if we use (again!) Schur lemma to claim that

$$
\left\|T_{0}\right\| \leq \frac{1}{\pi} \max _{|x| \leq 1} \int_{-1}^{1}\left|\frac{\sin (y-x)}{y-x}\right| d y:=B^{2} .
$$


A function $h(t)=\frac{\sin t}{t}$ is even, positive on $[-\pi, \pi]$ and monotone decreasing on $[0, \pi]$. Indeed,

$$
h^{\prime}(t)=\frac{t \cos t-\sin t}{t^{2}}=-\frac{1}{t^{2}} \int_{0}^{t} \tau \sin \tau d \tau<0,0<t \leq \pi .
$$

Therefore,

$$
\begin{aligned}
\frac{1}{\pi} \int_{-1}^{1} h(y-x) d y \leq \frac{2}{\pi} \int_{0}^{1} h(t) d y & <\frac{2}{\pi} \int_{0}^{1}\left(1-\frac{t^{2}}{6}+\frac{t^{4}}{120}\right) d t= \\
& =\frac{2}{\pi}\left(1-\frac{1}{18}+\frac{1}{600}\right)<.60232
\end{aligned}
$$

and

$$
b<B<.77608 \text { or } \frac{1-b}{2}>.11195>\frac{1}{9} .
$$

This completes the proof of Lemma 6 in the case $h=H=1$. General case for $h H=1$ immediately follows if we would use the following property of Fourier transforms:

$$
(\mathcal{F} g(r x))(s)=1 / r(\mathcal{F} g)(s / r) \text {. }
$$

\section{REFERENCES}

[Ca] Calderon, A.-P. Spaces between $L^{1}$ and $L^{\infty}$ and the theorem of Marcinkiewicz. Studia Math. 261966 273-299.

[DK] Harry Dym, Victor Katsnelson, Contributions of Issai Schur to Analysis, Studies in Memory of Issai Schur, Progr. in Math., 210, Birkhauser, Boston, MA, 2003 arXiv:0706.1868 Section 3.

[FS] Gerald B. Folland, Alladi Sitaram, The Uncertainty Principle: a Mathematical Survey, Journal of Fourier Analysis and Appl., vol. 3, No. 3 (1997), 207 - 238. (Section 7 and 8)

[HJ] V. Havin and B. Joericke, The Uncertainty Principle in Harmonic Analysis, 1994, Springer, Berlin.

[HS] Paul R. Halmos and Viakalathur Shankar Sunder, Bounded integral operators on $L^{2}$ spaces, Ergebnisse der Mathematik und ihrer Grenzgebiete (Results in Mathematics and Related Areas), vol. 96, Springer-Verlag, Berlin, 1978. Theorem 5.2.

[KMS] M. Kac, W. L. Murdock, and Szego, On the eigenvalues of certain Hermitian forms, J. Rational Mech. Anal., vol. 2 (1953), 767 - 800.

[KC1] Krotkov, Pavel; Chubukov, Andrey, Non-Fermi liquid and pairing in electron-doped cuprates, Physical Review Letters, 96, Issue 10 (March 17, 2006), pp. 107002 - 107005.

[KC2] Krotkov, Pavel; Chubukov, Andrey, Theory of non-Fermi liquid and pairing in electron-doped cuprates, Physical Review B, 74, Issue 1 (July 01, 2006), pp. 014509 - 014524.

[LP1] H. J. Landau and H. O. Pollack, Prolate spheroidal wave functions: Fourier analysis and uncertainty II, Bell Syst. Tech. J., 40 (1961), 65 - 84 
[LP2] H. J. Landau and H. O. Pollack, Prolate spheroidal wave functions: Fourier analysis and uncertainty III : the dimension of the space of essentially timeand band-limited signals, Bell Syst. Tech. J., 41 (1962), 1295 - 1336

[LV] Anatoly Larkin, Andrei Varlamov, Theory of Fluctations in Superconductors, Intern. Series of Monographs on Physics 127, Oxford Univ. Press, 2005

[Mi] Boris Mityagin, An interpolation theorem for modular spaces. (Russian) Mat. Sb. (N.S.) 66 (108) 1965 473-482. (English translation in Interpolation spaces and allied topics in analysis (Lund, 1983), Lecture Notes in Math., 1070, Springer, Berlin, 1984, 10-23.

[SP] D. Slepian and H. O. Pollak, Prolate spheroidal wave functions: Fourier analysis and uncertainty I, Bell Syst. Tech. J., 40 (1961), $43-63$

[Pa] S. V. Parter, On the extreme eigenvalues of truncated Toeplitz matrices, Bull. Amer. Math. Soc., vol. 67 (1961), $191-196$.

[Sc] I. Schur, Bemerkungen zur Theorie der Beschrankten Bilinearformen mit unendlich vielen Veranderlichten, J. Reine Angew. Math. 140 (1911), 1 - 28

[Wi1] Harold Widom, On the Eigenvalues of Certain Hermitian Operators,Trans. of the Amer. Math. Soc., vol. 88, No. 2 (July 1958), 491 - 522.

[Wi2] Harold Widom, Extreme Eigenvalues of Translation Kernels, Trans. of the Amer. Math. Soc., vol. 100, No. 2 (Aug. 1961), 252 - 262.

[Wi3] Harold Widom, Extreme Eigenvalues of N-dimensional convolutionoperators, Trans. of the Amer. Math. Soc., vol. 106 (1963), $391-414$

Department of Mathematics, The Ohio State University, 231 West

18Th Ave, Columbus, OH 43210, USA

E-mail address: mityagin.1@osu.edu 